How to cite this article:

Mohd Sharif, N. A., Ahmad, N., Ahmad, N., Mat Desa, W. L. H., Mohamed Helmy, K., Ang, W. C., \& Zainol Abidin, I. Z. (2019). A fuzzy rule-based expert system for asthma severity identification in emergency department. Journal of Information and Communication Technology, 18(4), 415-438.

\title{
A FUZZY RULE-BASED EXPERT SYSTEM FOR ASTHMA SEVERITY IDENTIFICATION IN EMERGENCY DEPARTMENT
}

\author{
${ }^{1}$ Nurul Atikah Mohd Sharif, ${ }^{1}$ Norazura Ahmad, ${ }^{1}$ Nazihah Ahmad, \\ ${ }^{1}$ Wan Laailatul Hanim Mat Desa, ${ }^{2}$ Khaled Mohamed Helmy, \\ ${ }^{3}$ Wei Chern Ang \& ${ }^{4}$ Ida Zaliza Zainol Abidin
}

${ }^{I}$ School of Quantitative Sciences, Universiti Utara Malaysia, Malaysia.

${ }^{2}$ Faculty of Medicine, AIMST University, Malaysia.

${ }^{3}$ Clinical Research Centre, Hospital Tuanku Fauziah, Malaysia.

${ }^{4}$ Emergency and Trauma Department, Hospital Tuanku Fauziah, Malaysia.

nurulatikah0791@gmail.com;norazura@uum.edu.my;nazihah@uum.edu. my;laailatul@uum.edu.my;kh_clinic@yahoo.com;wei.ang.1990@gmail.com; aizac2@yahoo.com

\begin{abstract}
The emergency department (ED) of a hospital is an important unit that deals with time-sensitive and life-threatening medical cases. Rapid treatment and accuracy in diagnosis are considered the main characteristics of excellent operational processes in ED. However, in reality, long waiting time and uncertainty in the diagnosis has affected the quality of ED services. Nonetheless, these problems can be improved by utilising computing technologies that assist medical professionals to make fast and accurate decisions. This paper investigates the issues of under-treatment and uncertainty condition of acute asthma cases in ED. A novel approach, known as the fuzzy logic principle is employed to determine the severity of acute asthma. The fuzzy set theory, known as Fuzzy Rule-based Expert System for Asthma Severity (FRESAS) determination is embedded into the expert system (ES) to assess the severity of asthma among patients in ED. The proposed fuzzy methodology effectively
\end{abstract}


manages the fuzziness of the patient's information data, and determines the subjective judgment of medical practitioners' level on eight criteria assessed in severity determination. Knowledge acquisition and representation, fuzzification, fuzzy inference engine, and defuzzification are the processes tested by the FRESAS development that incorporates expert advice. The system evaluation is performed by using datasets that were extracted from the ED clerking notes from one of the hospitals in Northern Peninsular Malaysia. System evaluation demonstrates that the proposed system performs efficiently in determining the severity of acute asthma. Furthermore, the proposed system offers opportunities for further research on other types of diseases in ED, and improves other hybridisation approaches.

Keywords: Emergency department, acute asthma, fuzzy rule-based.

\section{INTRODUCTION}

The emergency department (ED) is a unit in the hospital that assesses and treats unscheduled patients arriving for immediate treatment. Apart from conducting emergency treatments, accurate diagnosis is also a critical aspect of excellent services in the ED. With the evolution of computerised systems, the incorporation of an expert system would assist medical decision-making processes in ED. This paper focuses on the development of an expert system (ES) for asthma severity identification in the ED. The asthma cases were chosen as the medical domain to be investigated, instead of the many other cases at the ED as the boundaries of asthma studies are carefully identified. The adult acute asthma cases were selected to be incorporated into the ES due to its ability to fulfil the criteria needed for ES development, and the immediate need to obtain fast and accurate decisions in asthma management.

Asthma is a well-known disease that is described as a condition when both chronic airways are inflamed, while increasing airway hyper-responsiveness that leads to the narrowing of the airways, thickening of the airway wall and increase of mucus formation. It is usually observed through the typical symptoms such as wheezing, chest tightening and shortness of breath (Colledge, Walker \& Ralston, 2010). There are two types of asthma, which are acute asthma and chronic asthma. Acute asthma refers to an asthma attack that can last minutes or days, while chronic asthma is a lifetime disease. In addition, acute asthma is diagnosed as asthma flare-up, exacerbation, or an attack that is typically seen in the ED, that is usually diagnosed as chronic asthma if the symptoms are persistent. In managing acute asthma cases, determining the severity of asthma is essential to prescribing the suitable medicine and 
treatment. An incorrect acute asthma severity identification may lead to undertreatment, and would worsen the asthma. Therefore, the purpose of this paper is to develop a fuzzy rule-based expert system that is capable of determining the severity of asthma in the ED. The developed system may help the triage officer to hasten the diagnosing process, and eventually help the patient to obtain the correct treatment.

The subsequent sections will be thoroughly discussed in this paper. In the following sections, literature review and the methodology applied in the study are presented. The results from the proposed system are discussed, and a brief conclusion is provided.

\section{RELATED WORK}

\section{Asthma Diagnosis in Emergency Department}

Asthmatic patients assume that the ED is the only place to seek emergency treatment, instead of other primary care providers due to the easy access to ED (The National Healthcare Statistics Initiative, 2015). It has been reported that 1.8 million asthmatic patients have had emergency visits to ED every year in the United States, where female patients have had the highest visiting rate, from all age groups (Ginde, Espinola \& Camargo, 2008; Fernandes, 2015). In Europe, $23.9 \%$ of asthmatic patients were reported to have emergency care visits yearly (Price, Fletcher \& van der Molen, 2014). Furthermore, about 38\% of asthma patients in eight Asian countries including Malaysia had sought out asthma treatments in ED (Price et al., 2015). Respiratory cases are the most common emergency case chief complaints, that made up $40 \%$ of ED visitors, and has indicated that almost $34.6 \%$ of common diagnosis in ED is the acute exacerbation of bronchial asthma (Ginde, Espinola \& Camargo, 2008).

An ED is one of the most important department in a hospital that does not only receive asthma cases to be treated, but accepts various other diseases and emergency cases. Throughout the years, it has been reported that the number of ED visits had risen dramatically since 2009, from 7.143 million patients to 9.362 million patients in 2013 (The National Healthcare Statistics Initiative, 2015). The rise in cases and diagnoses over the years from high-acuity patients, and the increase in the number of patients in ED as led to several critical issues of poor quality service such as overcrowding, long waiting time, overutilisation of emergency service, and high variability and potential for medical error (Hitchcock, 2011; Hoot \& Aronsky, 2008; Ismail, Karim, Majid \& Saiful, 2009; Richardson \& Mountain, 2009). Hence, the increasing 
pressure exerted on health practitioners to deliver swift, efficient, and precise diagnosis, has forced them to find alternatives to streamline the delivery of high-quality medical care to their patients (Agrawal, \& Kosowsky, 2009).

Many issues at ED, such as long waiting time and the uncertainty conditions create an extremely high-pressure department that are required to perform speedy treatments and produce highly accurate diagnosis (Sinclair \& Croskerry, 2010; Ahmad, Ghani, Anton, Tahar \& Teo, 2012). Asthma exacerbation severity determination is an important procedure during the triage process in ED for the immediate treatment to reduce the possibility for the disease to worsen, and the delay for treatment may lead to death (Broaddus et al., 2016). Adherence to asthma management guideline in ED has shown improvement in clinical care (Scribano, Lerer, Kennedy, \& Cloutier, 2001). However, not all healthcare practitioners routinely adhere to asthma guideline recommendations, due to several limitations such as obstacles in organisations that lack protocol in the implementation strategy, and after all, belief and attitude of healthcare practitioners contribute to a major factor of guideline disobey (Wahabi \& Alziedan, 2012). In addition, a study has indicated that the implementation of a decision support system can be conducted to influence healthcare practitioners towards asthma guideline, rather than through feedback and auditing Okelo et al., (2013). During asthma severity identification, one of the challenges in asthma management is that the asthma is classified based on the historical symptom patterns and evidence of obstruction in variable expository airflow (Levy et al., 2009). The historical data of asthmatic information are gained from the caretaker and involves layman linguistic terms in describing the patient's condition. Consequently, this contributes to uncertainties on the information and possible misinterpretation of asthma conditions (Yang, Simons, Foty, Subbarao, To, \& Dell, 2016).

\section{Modelling Uncertainty in the Expert System}

In the case of uncertainty modelling approach, various mathematical approaches have been developed and introduced, such as the Fuzzy set theory (Zadeh, 1965), rough set theory (Pawlak, 1982) and Bayesian network (Spiegelhalter, Myles, Jones, \& Abrams, 1999). However, the fuzzy set theory is considered the best-suited method in handling linguistic uncertainties (Zadeh, 1972), vagueness, measurement imprecision, symptomatic remedies and natural diversity in the acute asthma severity knowledge (Steimann, 2001). In addition, the combination of a rule-based approach with fuzzy reasoning and knowledge information are utilised to examine the potential and possible areas for fuzzy rule-based ES implementation in medical diagnosis and identification (Innocent, John \& Garibaldi, 2004; Sikchi, Ali, \& Shkchi, 
2013; Siler \& Buckley, 2005; Zadeh, 1988). Moreover, the necessity of the fuzzification approach in medical diagnosis ES is to overcome the problem of capturing subjective or ambiguous evaluations that are made in medical diagnosis (Ali \& Singh, 2010). Additionally, the fuzzification approach leads to better outcomes compared to the conventional practice, whereby doctors in ED usually refer their patients to other experts, thus establishing the need for practical guidelines in decision making. The application of this ES system would assist in decision making processes as the expert knowledge has already been embedded into the system. The linguistic terms commonly used by patients to describe their condition are general and vague. Hence, the fuzzification approach is capable of converting the linguistic terms into valuable information for disease identification.

Historically, ES is a clause for Artificial Intelligence (AI) software that was established for real-world usage since the 1970s. However, the study of AI philosophy had started 2000 years ago with a variety of vicissitudes in the research field, where at last the first recognised ES, known as DENDRAL was invented by Edward Albert Feigenbaum in 1965 (Buchanan \& Feigenbaum, 1978; Leondes, 2002), and was subsequently followed by MYCIN (Shortliffe, 1976), XCON (McDermott, 1980) and many more. Since the introduction of these concept on a knowledge-based system or ES, various other ES were created. As ES is analogues to human behaviour on reasoning knowledge and problem-solving skills, this has led to a high demand of intelligent computer programs in diverse areas such as engineering, manufacturing, medicine, management, military, education, training, and many more (Feigenbaum, 1977; Tan, Wahidin, Khalil, Tamaldin, Hu, \& Rauterberg, 2016). In the field of security, various organizations have implemented ES to overcome several issues, such as shoplifting (Mohd Zahari \& Zaaba, 2017). With the extension of various methods and techniques that have been utilised, the medical field has provided the opportunity for the development of ES.

\section{Fuzzy Expert System for Diagnosing Asthma}

From literature, the utilisation of fuzzy rule-based method as a decision support system for disease diagnosis and identification were explored, and a comprehensive review has been conducted by several studies which indicated the extended use of this methodology in the medical area (Mishra, 2014; Mishra \& Jha, 2015; Seising, Schuh \& Adlassnig, 2003). The employment of fuzzy ES in the asthma case is common with several published papers, specifically in dealing with asthma cases that have started during pre-treatment of medical consultation till the predictions on the fatal causes of asthma. In 2007, Lurie and her team applied the fuzzy technique in assessing patient's perception on 
asthma severity, and compare them with the GINA 2006 guidelines (Lurie, Marsala, Hartley, Bouchon-Meunier \& Dusser, 2007).

In 2009, Computer-aided Intelligent Diagnostic System for Bronchial Asthma (CAIDSA) was designed and developed by a research team from India. It is the first interactive computer-aided intelligent diagnostic system for the investigation of the type of asthma evaluation and its degree of severity. The system performance obtained an accuracy of 90.03\% (Chakraborty, Mitra, Mukherjee \& Ray, 2009). However, this research implemented neural network approach, where the hidden layers are unable to provide a clear explanation on the choice of answers that were made. In clinical practise, every decision taken should be deeply understood and presented with strong evidences. On the other hand, a group of researchers had utilised the Fuzzy Rule-based Expert System (FRES) to determine the degree of possibility of asthma at initial stages, where the results suggested a 94\% sensitivity and 100\% specificity in 2010 . In addition, the authors modified the FRES approach according to a different scope of study that predicted fatal asthma by determining the degree of asthma instability. However, more data was needed to validate the performance of their model. In 2012, the authors extended the implementation of FRES in classifying asthma diagnosis, evaluation, management, communicative facilities and prediction to assess the level of asthma exacerbation. Furthermore, the same approach was implemented to determine the level of asthma control with main features in order to decrease the cost of examination in asthma therapy processes (Zarandi, Zolnoori, Moin, \& Heidarnejad, 2010; Zolnoori, Zarandi, Moin \& Taherian, 2012; Zolnoori, Hossein, Zarandi \& Moin, 2010; Zolnoori, Zarandi \& Moin, 2012). Later, a team of Indian researchers had developed and implemented an ES for detecting and estimating diseases that are related to the lungs. The system distinguished patients based on their symptoms and performed diagnosis with the appropriate pulmonary disease, which were either asthma, tuberculosis or chronic obstructive pulmonary disease (COPD) (Anand, Kalpana, Vijayalakshmi, Hartley \& Boucho-meunier, 2013).

Most of the aforementioned studies in the fuzzy ES field were conducted in different countries, however, limited studies have been carried out in the local context, especially in Malaysia. Although some researches have conducted studies on asthma severity, no studies have been found that implemented the full criteria suggested by the current guideline, which makes the emergency department of hospitals the perfect setting for system evaluation. To the best of our knowledge, this study is the first in Malaysia that applies the GINA 2017 guideline into a computerised system. To implement an ES in ED, the system must possess the ability to be a fast decision support system that can 
handle uncertain conditions. There are many suitable methods to develop an ES, however for the acute asthma problems, the rule-based deduction method is considered, due to the following existing criteria of the case problem:

i. The main requirement for the application of this method is that the underlying knowledge must be organised, whereby the asthma severity identification has been inputted by an authorized health organization with the corresponding guidelines.

ii. The classification of asthma severity are classified in distinct categories, where a table format guideline to determine the asthma severity can be referred from Global Initiatives for Asthma (GINA) 2017.

iii. In order to assess the asthma severity, small context dependence is employed as one of the criteria that is applied into the rule-based method in ES.

iv. Rules are the universal knowledge representation technique that are applied in ES development (Tripathi, 2011).

The key contribution of this paper is the development of a virtual system that implements the current asthma guidelines to initiate early treatment procedures on patient's asthma condition. The proposed approach is applied during the triage process when there are usually no medical doctors allocated at this stage. Thus, the system would assist medical assistants to manage the asthmatic patients correctly based on the guidelines embedded in this expert system. Hence, this would improve the system management, mainly on time management and the handling of uncertain issues.

\section{METHODOLOGY}

This section discusses the research framework that was adapted from the Expert System Development Life Cycle (ESDLC) (Liebowitz, 1997). This section is structured as follows: definition of knowledge acquisition and representation, procedures carried out for model development, and model evaluation.

\section{Knowledge Acquisition and Representation}

Knowledge Acquisition (KA) is a process of extracting, structuring and synchronising knowledge that were gathered from various sources such as by experts in the domain of interest, textbooks, technical papers, databases, reports and the environment. The process of KA on acute asthma information began with self-reading on the issues of asthma by researching information 
from the internet, as well as referring to medical books and journals. The objective of self-reading is to identify key areas and examine case studies on the acute asthma cases, and to obtain a basic understanding of the medical terms used in asthma cases.

Subsequently, a semi-structured interview method was applied with multiple interview sessions with three academicians that specialised in mathematics and expert system field, one respiratory specialist, one clinical researcher and the Head of Emergency Department from one of the hospitals in Northern Malaysia. This group of people are called domain experts. The semistructured interview questions were designed based on the information gained through self-reading, that included an open-ended question regarding asthma diagnosis and management. The first meeting was conducted to identify the common practices in handling acute asthma cases in local ED and determine the most significant assessment used in the classification of asthma severity. Several local association and guideline in managing acute asthma have been introduced such as the Global Initiative for Asthma (GINA), Asthma Council Malaysia (ACM) and Clinical Practice Guideline (CPG) for asthma management. The information gathered from the first meeting was analysed extensively to determine its correlation with the self-reading information. This process is conducted to ensure the originality and validity of the knowledge that are implemented into the expert system. Further meetings were organised to refine the case problem and reexamine the rules that were briefly constructed at the first meeting. After a lengthy discussion, a list of eight input parameters were decided to be used in the model development for Fuzzy Rule-based Expert System for Asthma Severity determination (FRESAS) based on the main GINA guidelines, whereby the output parameter diagnoses the severity of acute asthma as either moderate or severe acute asthma.

On the other hand, Knowledge Representation (KR) is the process of knowledge transformation that have been verified and gained through knowledge acquisition, which produces information that are computable. The knowledge gathered is presented in the form of a mathematical formulation. The mathematical formulation for this FRESAS model consists of fuzzy sets, membership function (MF), and fuzzy rule-based engine. A fuzzy set is a pair that are denoted as $\left(\mathrm{A}, \mu_{\mathrm{A}}\right)$, where $\mathrm{A}$ is a set of all input variables.

\section{Model Development}

Model development is a significant part of this study. FRESAS is developed based on two integrated techniques, which are fuzzy set theory and rule- 
based ES. In the fuzzy-based approach, Figure 1 shows the components of the proposed FRESAS method that consists of a fuzzy knowledge base, an inference engine that includes fuzzification, fuzzy inference rule, and defuzzification (working memory and user interface). Each of the FRESAS component development are elaborated in the next section.

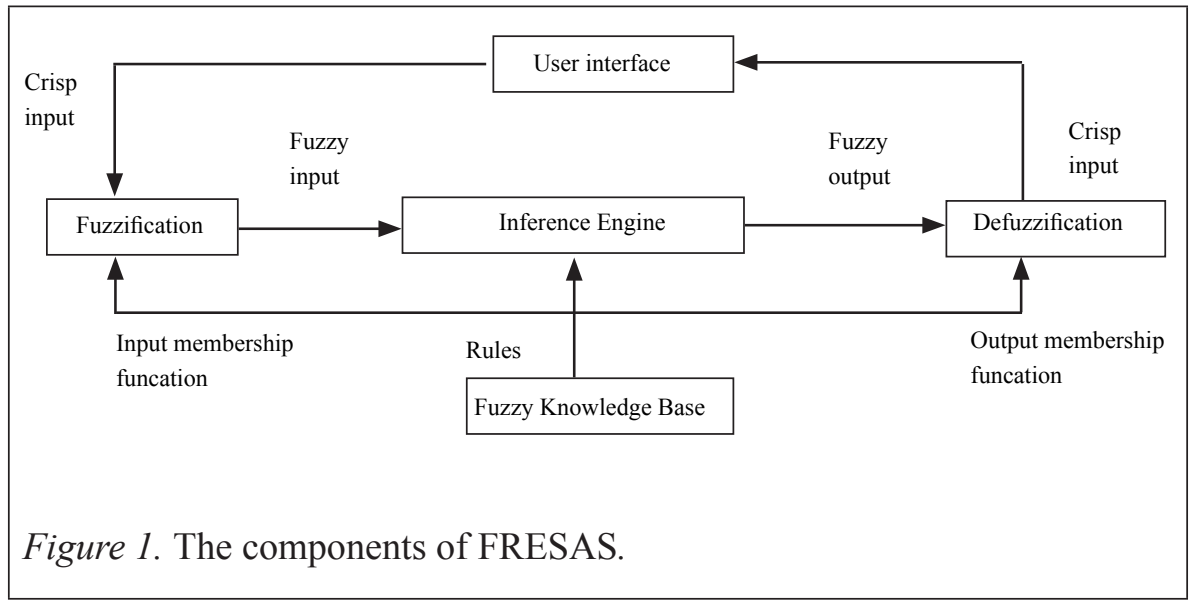

\section{System Inputs Variable}

Based on the knowledge acquisition process, there are a total of 13 inputs, where eight main input parameters were considered for the fuzzy system and five additional compulsory inputs were assessed to fulfil the final value of Peak Expiratory Flow (PEF). Through the inclusion of the PEF variable, in common medical practice, the normal PEF value was analysed from the current EU standard (EN 13826) PEF graph, and the resulting value obtained was compared to the measured peak flow meter. In current practice, the percentage of PEF is calculated manually however, FRESAS provides a support system that calculates the PEF value automatically based on the individual patient criteria. Figure 2 shows the relationship of input variables between fuzzy model inputs and the necessity for additional system inputs.

\section{Fuzzy Knowledge Base}

The fuzzy knowledge base of FRESAS is developed with the help of the medical experts' past experiences as well as references to the current asthma guideline. The preliminary rules were formed and verified by the expert, and if there were any conflicts, it was resolved by a consensus. The final rules were written in the format of $<$ IF (antecedent) THEN (consequent) $>$. An example of the resulting rule statement is as follows: 
If (Alertness is Absent) and (Talk is Phrases) and (Auscultation is Audible Wheeze) and (Sit is Sits Up) and (Accessory Muscle is No) and (PEF is $>50$ (low risk)) and (PR is 100-120(medium)) and (SpO2 is >90) and (RR is Increased) then (Asthma Severity is Mild) (1)

When the previous condition is satisfied, then the rule is triggered and the action of the consequent is performed. A total of 216 rules statement for FRESAS were constructed.

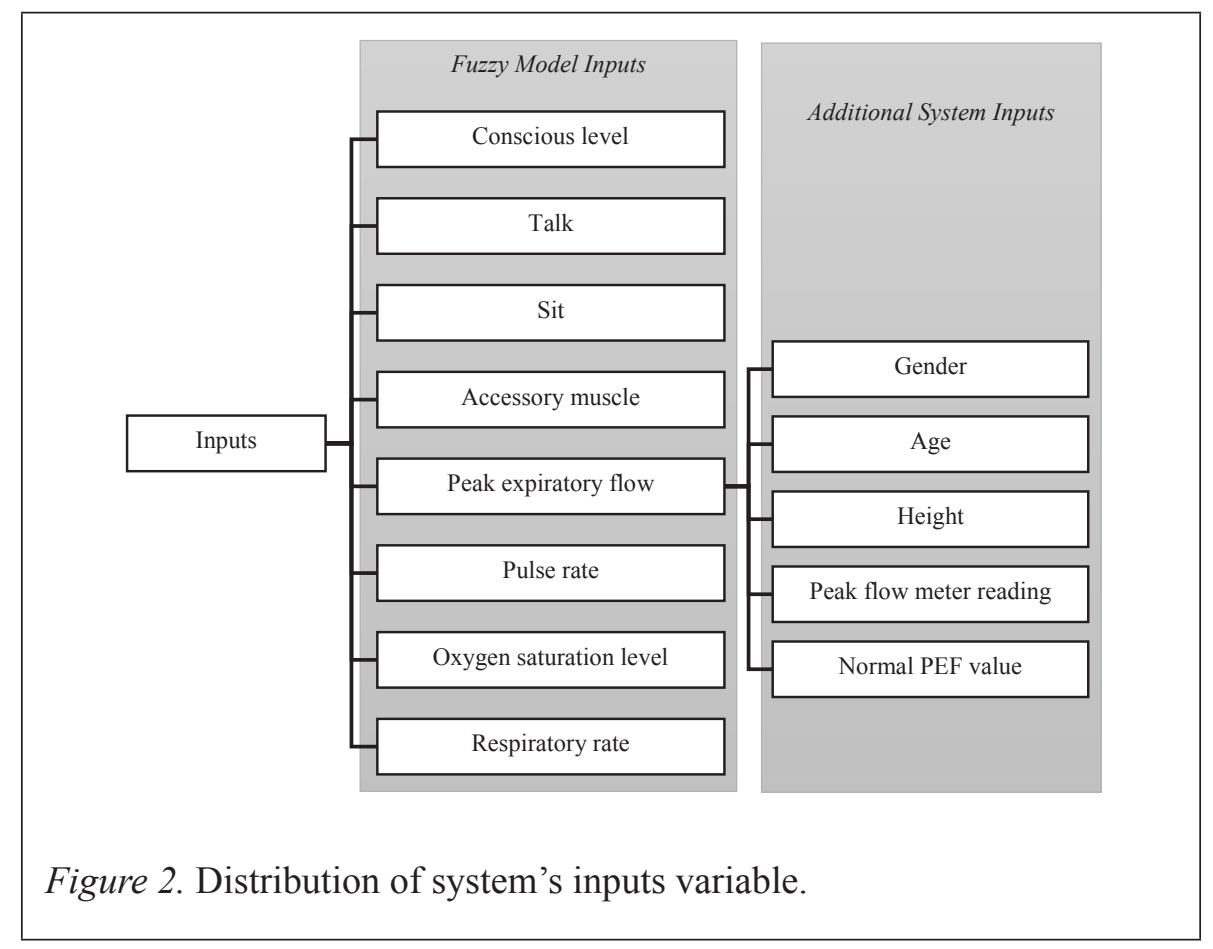

\section{Fuzzification Process}

During the fuzzification process, the real-valued on eight inputs of information are transformed into a membership grade of the fuzzy sets. The input parameters are considered as a fuzzy variable or fuzzy set. The fuzzification process determines the linguistic variable and membership function of each fuzzy set. A linguistic variable is the value determined on the words or sentences uttered naturally. In general, the linguistic variable is of proportional nature, whereas fuzzy set constitutes the linguistic variables in fractional values, between the ranges of 0 to 1 . In this study, the linguistic variables for each fuzzy variables are shown below. 
Conscious level $\xi\{$ Not agitated,Agitated $\}$

Talk $\xi\{$ Phrases, Words\}

Sit $\xi\{$ Prefer to sit, Sits hunched forwards\}

Accessory muscle $\xi$ \{Not used,Being used\}

PEF $\xi\{$ Low risk, High risk\}

Pulse rate $\xi$ \{Low risk, High risk\}

Oxygen saturation level $\xi$ \{Low risk, High risk\}

Respiratory rate $\xi$ \{ncreased,High risk\}

The domain expert defines these linguistic variables to coincide with the medical terms used. Each fuzzy variable has its universe of discourse presented in various interval range value. Among the various membership function, the trapezoidal membership function was employed in this system due to its generality and capacity to contain more fuzzy information. Furthermore, due to the natural description of certain fuzzy variables such as oxygen saturation levels for which the values are read in a range, the trapezoidal graph was determined to be the most suitable representation for oxygen saturation levels in the fuzzy model. The identification of the memberships of each linguistic variable for each input variables were then transferred into the Fuzzy Logic Designer toolbox in Matlab. Figure 3 to Figure 10 depict the membership function graphs for the eight (8) inputs.
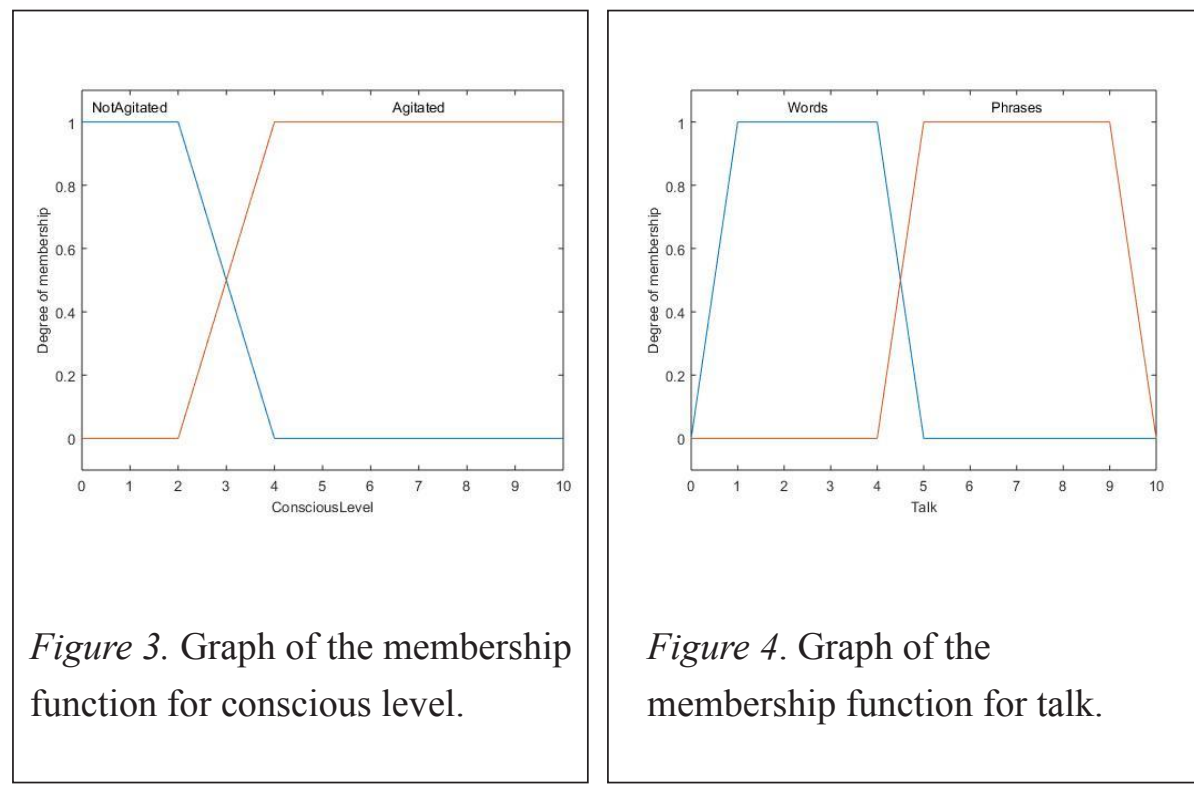

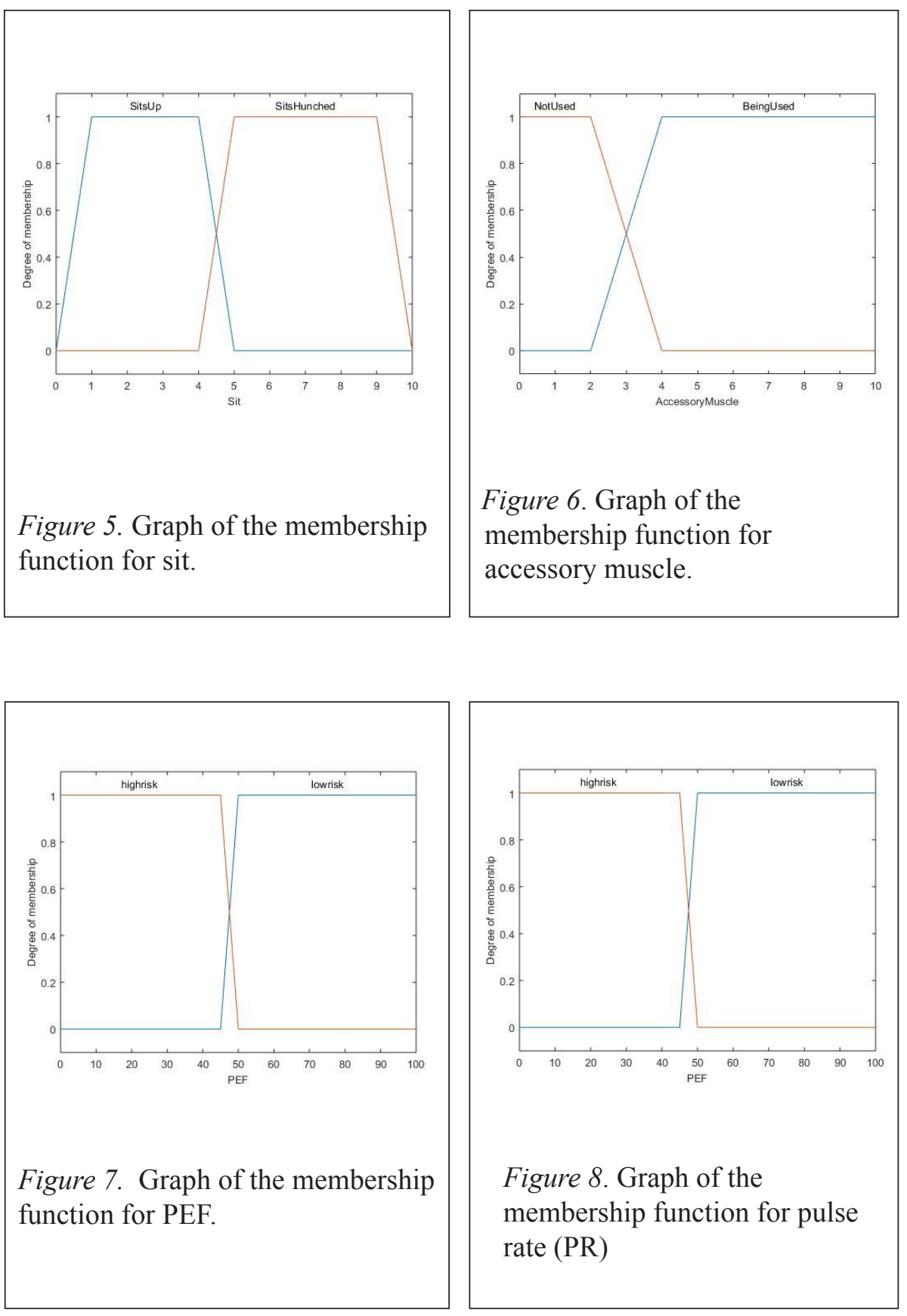

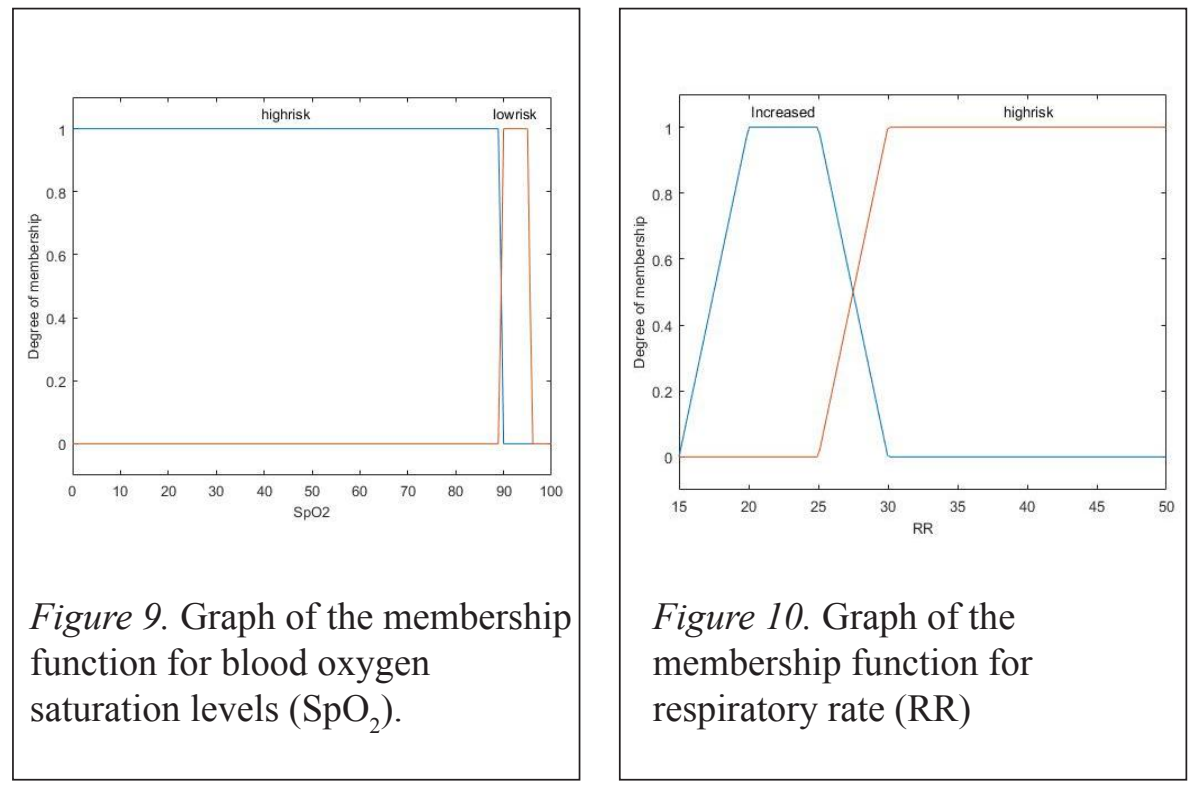

An example of the fuzzy membership expression for conscious level is represented in Equation 1.

Conscious level input;

$$
\begin{gathered}
\mu_{\text {Not Agitated }}(x)=\left\{\begin{aligned}
1, & x<2 \\
\frac{4-x}{2}, & 2 \leq x<4
\end{aligned}\right. \\
\mu_{\text {Agitated }}(x)=\left\{\begin{aligned}
\frac{x-2}{2}, & 2<x<4 \\
1, & x \geq 4
\end{aligned}\right.
\end{gathered}
$$

At this stage, the system identifies the linguistic variable selected in the user interface and matches it with the $x$-axis of the membership function graph of the fuzzy inference system. The value from the $x$-axis of the membership function graph is then mapped on the $y$-axis to obtain the fuzzification value, i.e. the confident level of the linguistic variable chosen. The results of the fuzzification outputs will then undergo the fuzzy logic operation process.

\section{Fuzzy Inference Engine}

In general, the inference engine is used to make an inference or reasoning. This method determines the rules that are needed for selection, by matching the 
facts from the fuzzification process output and fuzzy knowledge base which are organized based on priority, and then executed. The Mamdani-style was implemented due to its ability to present humanistic experiences thoroughly and illustrates the knowledge from a broader perspective.

In a part of the inference engine, there is a fuzzy operator which is responsible for combining the input of the fuzzy system. There is more than one input to be combined with the antecedent part of IF-Then rules. Hence, the fuzzy operator applied is based on the multivalued logic specifically focusing on min operation where it will present only one value that represents the antecedent part of the rule. The intersection of the fuzzy set min operation for FRESAS is presented in equation (2).

Let $\mu_{A} \ldots \mu_{H}$ be the membership functions that define the fuzzy sets $A$ until $H$, respectively on the universe X. $A$ until $H$ denotes the eight fuzzy inputs. The intersection of fuzzy sets $A$ until $H$ is a fuzzy set defined by the membership function below:

$$
\begin{aligned}
\mu_{A \cap B \cap \cap \cap D \cap E \cap F \cap G \cap H}(x) \\
\quad=\operatorname{Min}\left(\mu_{A}(x), \mu_{B}(x), \mu_{C}(x), \mu_{D}(x), \mu_{E}(x), \mu_{F}(x), \mu_{G}(x), \mu_{H}(x)\right)
\end{aligned}
$$

The result of the fuzzy operation determines the single output for antecedent, and continues to evaluate the consequent part of the overall rule statement.

The implication is a process to obtain an output from the following IF-THEN rule, after receiving outputs from the antecedent part as an implication input. Min method is used for the implication of the latter part which decreases the fuzzy set output. The implication is also applied to each of the rules in FRESAS. Therefore, in the process to defuzzify the results of FRESAS, the aggregation process is assigned to combine each output of the fuzzy set rule into a single fuzzy set. The input for the aggregation process is the list of the decreased output functions that are returned from the implication process for each of the rules. Subsequently, FRESAS implemented a sum built-up method for the aggregation procedure.

\section{Defuzzification Process}

As the aggregation output is defined within a range value, the defuzzification process is required to resolve the single number into a model output. FRESAS applied a widely utilized defuzzification method known as centroid, which 
computes the centre of the area under the curve into a single number. Mathematically, this centre of gravity (COG) is expressed as follows:

$$
C O G=\frac{\int_{a}^{b} \mu_{A}(x) x d x}{\int_{a}^{b} \mu_{A}(x) x d x}
$$

Let $A$ be the point representing the COG of the fuzzy set, and $a b$ is the interval of the graph. The end output is represented in real value and is used to present the user output. Figure 11 shows the graph of fuzzy output, where the real value extracted from the graph and the COG formula is applied.

The linguistic variable and equation that represent the FRESAS output are as follow:

$$
\begin{gathered}
\mu_{\text {Moderate }}(x)=\left\{\begin{aligned}
1, & 0<x<4 \\
\frac{5-x}{1}, & 4 \leq x \leq 5 \\
0, & 5<x<10
\end{aligned}\right. \\
\mu_{\text {Severe }}(x)=\left\{\begin{aligned}
0, & x<4 \\
\frac{x-4}{1}, & 4 \leq x<5 \\
1, & x>5
\end{aligned}\right.
\end{gathered}
$$

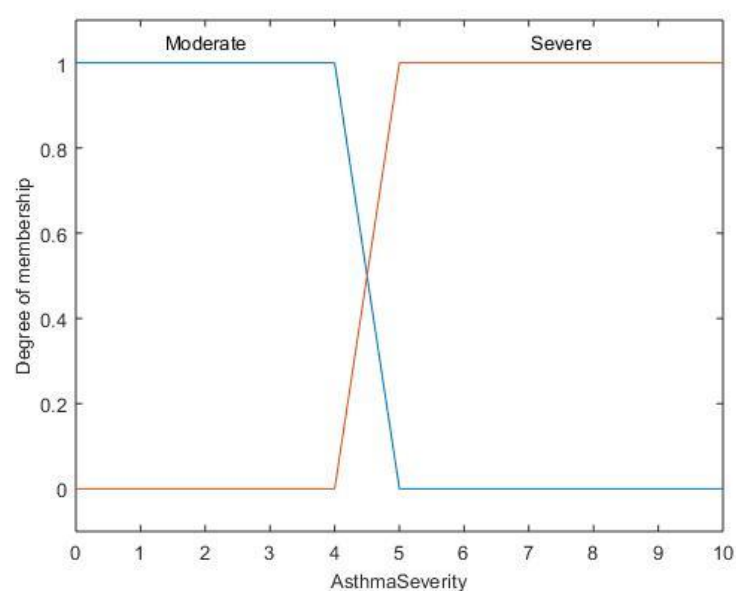

Figure 11. Graph of the Membership Function for Asthma Severity. 
To ensure the model is comprehensive and useful, a visual interface was developed. The visual interface system was programmed with App designer 2.0 tool in Matlab. Figure 12 shows the user interface of FRESAS used for the proposed approach. After consultation with the experts, the patients are required to fill up all the items in the interface to obtain a valid result. The constructed interface is composed of three zones; Zone 1 shows the input variable for patients personal information, Zone 2 shows the input variable for the patient symptoms and health data, and Zone 3 yields the asthma severity diagnosis output.

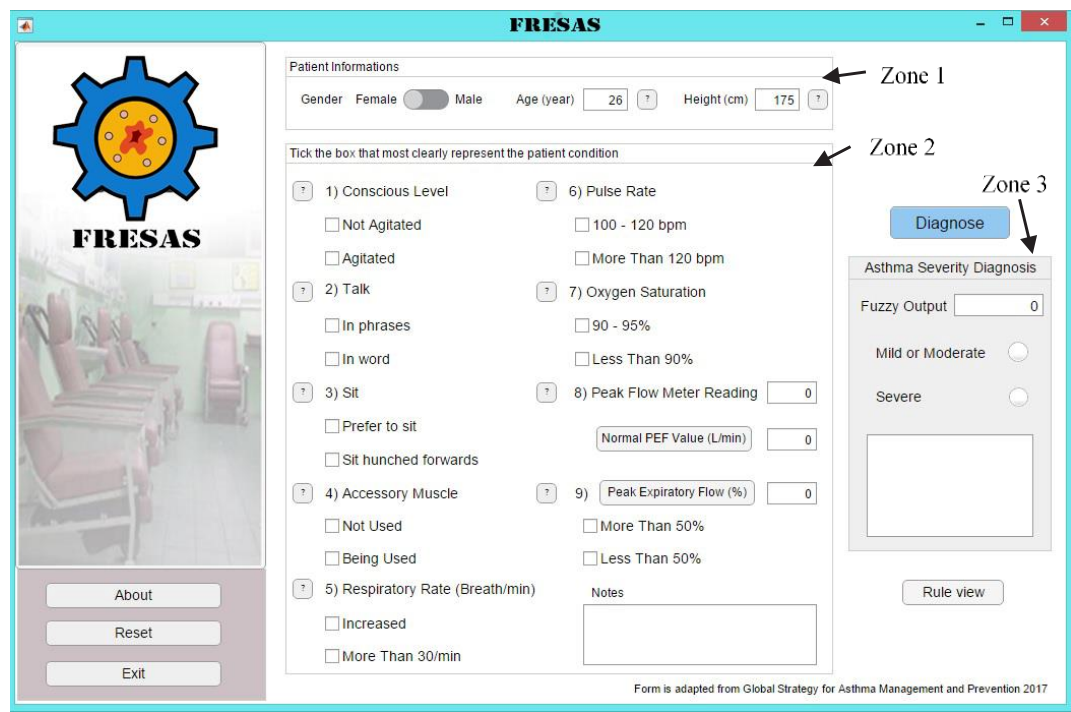

Figure 12. The User Interface of FRESAS.

\section{Model Evaluation}

To evaluate the applicability of FRESAS, model verification and validation was performed. For model verification, a series of expert consultation sessions were conducted to ensure that the knowledge inputted into the model would accurately represent the problem domain. On the other hand, for the model validation, 30 real cases were collected within a month, and used as evaluation data to validate the application and functionality of the model. This study is registered with the National Medical Research Registry, Malaysia (NMRR16-2729-32879) and was given approval by the Medical Research Ethics Committee (MREC), Malaysia. 


\section{RESULTS AND DISCUSSIONS}

All 30 data were used to determine the performance of FRESAS. From the data collected, $57 \%$ of the subjects are female while $43 \%$ are male. Figure 13 shows the number of male subjects based on the acute asthma severity with different diagnosis task. Both FRESAS and doctors' diagnosis were compared to the GINA guideline. The results indicated that FRESAS was able to diagnose the same number of subjects, in line with the guideline. On the contrary, the doctors' diagnosis task revealed over-diagnosis on moderate acute, and under-diagnosis on severe acute asthma severity. With the female subjects, FRESAS had prescribed the same diagnosis that are in line with the guidelines. However, the doctors' diagnosis resulted in over-diagnosis on moderate acute asthma severity, and under-diagnosis on severe acute asthma severity (Figure 14).
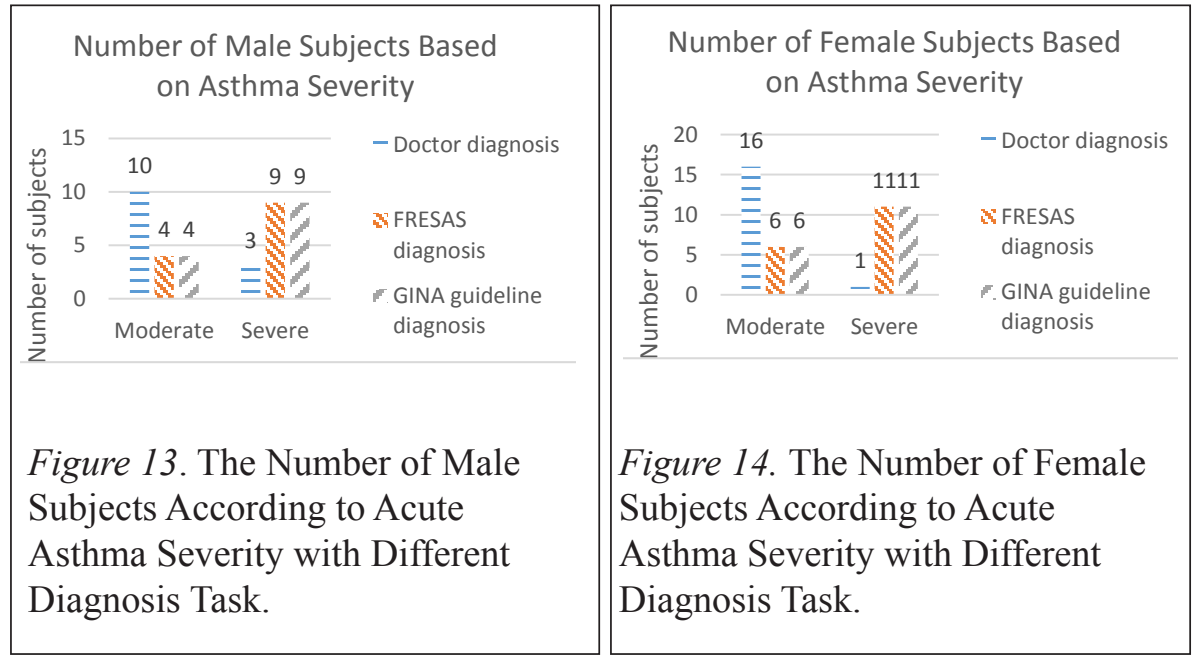

In this study, Cohen's kappa was used to test the agreement between the two groups, which are:

1. Group 1: Between the FRESAS diagnosis and GINA guideline diagnosis.

2. Group 2: Between the doctor's diagnosis and GINA guideline diagnosis.

GINA guideline is the gold standard for asthma severity diagnosis, and was established as the principal guideline for asthma management in ED. For Group 1, the kappa result is 1.0, which indicates that the level of agreement between FRESAS and GINA guideline to identify asthma severity cases is 
in perfect agreement. In Group 2, the kappa analysis output is 0.143 , which indicates that there is no level of agreement between the doctor's diagnosis and GINA guideline. Hence, the kappa analysis suggests that, with regards to the GINA guidelines, FRESAS has a system reliability of $100 \%$ as compared to doctor's diagnosis which only has a reliability of 14\%. The kappa results of Group 1 and Group 2 are shown in Table 2 and Table 3 respectively.

Table 2

The kappa result for group 1

\begin{tabular}{lccc}
\hline & Value & Approximate Significance \\
\hline Measure of Agreement & Kappa & 1.000 & .000 \\
$\mathrm{~N}$ of Valid Cases & 30 & \\
\hline
\end{tabular}

Table 3

The kappa result for group 2

\begin{tabular}{|c|c|c|c|}
\hline & & Value & Approximate Significance \\
\hline Measure of Agreement & Kappa & .143 & .129 \\
\hline $\mathrm{N}$ of Valid Cases & & 30 & \\
\hline
\end{tabular}

The aim of this study study is to design an expert system for acute asthma severity determination according to GINA 2017 guideline in the emergency department. The accurate evaluation in acute asthma severity has played a major role in medicine prescription accuracy that has subsequently led to more controllable asthma cases. The disregard of some variables in estimating asthma severity by doctors, inherent uncertainty of variables, and prescription based on restrictive categories are the main reasons for the underevaluation of asthma. The developed FRESAS was evaluated with real data of acute asthma obtained from the emergency department's medical records to measure the performance and effectiveness of the system. The results of the developed approach suggests that the built expert system of FRESAS is able to provide reliable and accurate diagnosis and hence, acceptable for real-time implementation.

The performance of FRESAS are consistent with other asthma ES based on GINA guideline such as CIADSA (Chakraborty et al., 2009). The findings obtained in this study are similar to previous studies that have examined the 
sensitivity and specificity of the expert system reported by Zarandi et al. (2010). The result of this research also matches those observed in an earlier study by Zolnoori et al. (2012) where the kappa result is one (1), indicating the agreement between the two comparison groups. In addition, a study suggested that the use of a computer decision support system for asthma was ineffective in improving patient outcomes, as the systems was rarely implemented by doctors and the low compliance to the advise generated by the system. Furthermore, the study further suggested that if the system was utilised comprehensively, the clinical outcomes may have improved (Matui, Wyatt, Pinnock, Sheikh \& McLean, 2014).

This study did not investigate the effectiveness of the FRESAS used for doctors to improve patient outcomes. Additionally, this study focused on the ability of FRESAS in determining acute asthma severity classification, instead of doctors compliance to utilise this expert system. Further research is required to investigate the behaviour and compliance of doctors to use computerized decision support systems, with regards to disease management.

Furthermore, the developed expert system was not intended to replace doctors, but was proposed to aid and support doctors, especially junior doctors to determine the severity of asthma and forecasting patient's health status. Based on the analysis done, doctors were reported to be less accurate in diagnosing acute asthma based on GINA guidelines and indicated that the adherence to asthma guideline is low among doctors, leading to discrepancies in diagnosis (Wisnivesky et al., 2008; Wahabi \& Alziedan, 2012). However, the low adherence of the GINA guidelines may be due to the changes made based on clinical experiences of the patient's current condition. In addition, the misleading diagnosis of doctors from GINA guidelines may not represent the competency of doctors, but rather the different practices that are not parallel to global guidelines, such as those employed in the Malaysian ED. The obtained results is subjected to the discretion of hospital ED management to initiate a suitable action for system improvements in determining asthma severity. Moreover, the introduction of the FRESAS diagnostic system is highly recommended for real-time application due to its ability to determine the severity of acute asthma in ED that strictly adheres to the GINA guidelines. This system can be applied in primary care settings, and in the general hospital practices as a standalone system or alongside other clinical systems. This approach can be used in medical research for assessing the effect of various risk factors on acute asthma severity levels and comparing the control statuses for asthmatic patients. 


\section{CONCLUSION}

The application of an expert system in diagnosing diseases in the ED is essential due to the special characteristic of ED that deals with time-sensitive and broad spectrum of life threatening cases. This study proposed an uncertainty model framework, known as a fuzzy rule-based ES. A total of 30 adult asthmatic patients in ED participated in this study to evaluate the fuzzy rule-based expert system for asthma severity identification (FRESAS). The system performance was analyzed by using Cohen's Kappa analysis. From the analysis, it was interpreted that FRESAS obtained a 100\% system reliability, while doctors' diagnosis obtained a 14\% diagnosis reliability based on the GINA guidelines. Hence, FRESAS system diagnosis is successful in differentiating moderate and severe acute asthma severity in adherence to the GINA guidelines, as compared to the doctors' diagnosis. Several limitations were identified and would require further study before the system can be fully utilized in the real world. Further experimentation of the system is still required to determine the validity of the system, and to obtain precise and fast outputs that ensure time management and system acceptance among medical practitioners.

\section{ACKNOWLEDGEMENT}

The authors wish to thank Universiti Utara Malaysia for their help and financial support in Geran Penjanaan (S/O code:13410).

\section{REFERENCES}

Agrawal, P., \& Kosowsky, J. M. (2009). Clinical practice guidelines in the emergency department. Emergency Medicine Clinics of North America, 27(4), pp. 555-567. doi. 10.1016/j.emc.2009.07.001.

Ahmad, N., Ghani, N. A., Anton, A. K., Tahar, R. M., \& Teo, A. H. (2012). Evaluating emergency department resource capacity using simulation. Modern Applied Science, 6(11), pp. 9-19. doi. 10.5539/mas.v6n11p9

Ali, Z., \& Singh, V. (2010). Potentials of fuzzy logic: an approach to handle imprecise data. International Journal of Engineering Science and Technology, 2(4), pp. 358-361. doi. 10.1.1.188.7835

Anand, S. K., Kalpana, R., Vijayalakshmi, S., Hartley, S., \& Boucho-meunier, B. (2013). Design and Implementation of a fuzzy expert system for detecting and estimating the level of asthma and chronic obstructive pulmonary disease. World Applied Sciences Journal, 23(2), pp. 213223. doi: 10.5829/idosi.mejsr.2013.14.11.73192 
Broaddus, V. C., Mason, R. J., Ernst, J. D., King, T. E., Lazarus, S. C., Murray, J. F., ... Gotway, M. (2016). Murray \& Nadel's Textbook of Respiratory Medicine (6th ed.). Elsevier Saunders. doi.org/10.1513/ AnnalsATS.201504-2510T

Buchanan, B. G., \& Feigenbaum, E. A. (1978). DENDRAL and MetaDENDRAL: Their applications dimension. Artificial Intelligence, 11(3), pp. 5-24. doi.org/10.1016/0004-3702(78)90010-3

Chakraborty, C., Mitra, T., Mukherjee, A., \& Ray, A. K. (2009). CAIDSA: Computer-aided intelligent diagnostic system for bronchial asthma. Expert Systems with Applications, 36(3), pp. 4958-4966. doi. 10.1016/j. eswa.2008.06.025

Colledge, N. R., Walker, B. R., \& Ralston, S. H. (2010). Respiratory disease. In Davidson's Principle and Practice of Medicine (21st ed, pp. 662672). Elsevier.

Feigenbaum, E. A. (1977). The art of artificial intelligence: Themes and case studies of knowledge engineering. International Joint Conference on Artificial Intelligent, 5(1014-1029), pp. 227-240. doi.10.21236/ ada046289

Fernandes, J. (2015). Asthma Emergency Department Visits : Asthma ED Visit Trends (Vol. 2951). Helena, Montana. doi.org/10.1378/ chest.120.4.1058

Ginde, A. A., Espinola, J. A., \& Camargo, C. A. (2008). Improved overall trends but persistent racial disparities in emergency department visits for acute asthma, 1993-2005. Journal of Allergy and Clinical Immunology, 122(2), pp. 313-318. doi. 10.1016/j.jaci.2008.04.024.

Hitchcock, R. (2011). Top 5 Benefits of Clinical Decision Support in the ED. Healthcare IT News. Retrieved from https://www.healthcareitnews. $\mathrm{com} / \mathrm{blog} /$ top-5-benefits-clinical-decision-support-ed

Hoot, N. R., \& Aronsky, D. (2008). Systematic review of emergency department crowding: causes, effects, and solutions. Annals of Emergency Medicine, 52(2). doi: 10.1016/j.annemergmed.2008.03.014 Innocent, P. R., John, R. I., \& Garibaldi, J. M. (2004). Fuzzy methods for medical diagnosis. Journal Application Artificial Intelligence, 19(1), pp. 69-98. doi.org/10.1080/08839510590887414

Ismail, N., Karim, Z. I. A., Majid, N., \& Saiful, H. J. (2009). Frequency of admittance and probability of inpatient treatment : experience of emergency department, Hospital Universiti Kebangsaan Malaysia. In Proceedings of the 13th WSEAS International Conference on Applied Mathematics (Math'08), 8, pp. 76-87.

Leondes, C. T. (2002). Expert systems: the technology of knowledge management and decision making for the 21 st century. Elsevier Inc. doi.10.14434/ijdl.v5i2.12891 
Levy, M. L., Quanjer, P. H., Booker, R., Cooper, B. G., Holmes, S., \& Small, I. R. (2009). Diagnostic spirometry in primary care: Proposed standards for general practice compliant with American Thoracic Society and European Respiratory Society recommendations. Primary Care Respiratory Journal, 18(3), pp. 130-147. doi: 10.4104/pcrj.2009.00054.

Liebowitz, J. (1997). The handbook of applied expert systems. Expert Systems. CRC Press LLC. Retrieved from http://portal.acm.org/ citation.cfm? $\mathrm{id}=524135$

Lurie, A., Marsala, C., Hartley, S., Bouchon-Meunier, B., \& Dusser, D. (2007). Patients' perception of asthma severity. Respiratory Medicine, 101(10), pp. 2145-2152. doi.org/10.1016/j.rmed.2007.05.027

Matui, P., Wyatt, J. C., Pinnock, H., Sheikh, A., \& McLean, S.. (2014). Computer decision support systems for asthma: a systematic review. Nature Partner Journal, Primary Care Respiratory Medicine, 24, 14005. doi: 10.1038/npjpcrm.2014.5

McDermott, J. P. (1980). RI: an expert in the computer systems domain. In Proceedings of the 1st Annual National Conference on Artificial Intelligence. Stanford University, August 18-21, pp. 269-271.

Mishra, N. (2014). A review on the application of fuzzy expert system for disease diagnosis. International Journal of Advanced Research in Engineering and Applied Sciences, 3(12), pp. 28-43.

Mishra, N., \& Jha, P. (2015). A survey on fuzzy medical expert system. International Journal of Computer Application, 1(5), pp. 84-90.

Mohd Zahari, M. K., Zaaba, Z. F. (2017). Intelligent responsive indoor system (IRIS): A Potential shoplifter security alert system. Journal of Information and Communication Technology, 16(2), pp. 262-282.

Okelo, S. O., Butz, A. M., Sharma, R., Diette, G. B., Pitts, S. I., King, T. M., ... Robinson, K. A. (2013). Interventions to modify health care provider adherence to asthma guidelines: a systematic review. Pediatrics, 132(3), pp. 517-534. doi: 10.1542/peds.2013-0779.

Pawlak, Z. (1982). Rough Sets. International Journal of Computer and Information Sciences, 11(5), pp.341-356. doi.org/10.1007/BF01001956

Price, D., David-Wang, A., Cho, S.-H., Ho, J. C.-M., Jeong, J.-W., Liam, C.K., ... Neira, G. (2015). Time for a new language for asthma control: results from REALISE Asia. Journal of Asthma and Allergy, 8, pp. 93-103. doi: 10.2147/JAA.S82633.

Price, D., Fletcher, M., \& van der Molen, T. (2014). Asthma control and management in 8,000 European patients: the REcognise Asthma and LInk to Symptoms and Experience (REALISE) survey. Primary Care Respiratory Medicine, 24, pp. 1-10. doi: 10.1038/npjpcrm.2014.9. 
Richardson, D. B., \& Mountain, D. (2009). Myths versus facts in emergency department overcrowding and hospital access block. Medical Journal of Australia, 190(7), pp. 369-374. doi: 10.5694/j.1326-5377.2009. tb02451.x

Scribano, P. V., Lerer, T., Kennedy, D., \& Cloutier, M. M. (2001). Provider adherence to a clinical practice guideline for acute asthma in a pediatric emergency department. Academic Emergency Medicine: Official Journal of the Society for Academic Emergency Medicine, 8(12), pp. 1147-52. doi.10.1111/j.1553-2712.2001.tb01131.x

Seising, R., Schuh, C., \& Adlassnig, K.-P. (2004). Historical topics on fuzzy set theory in biomedical engineering. Advances in Scattering and Biomedical Engineering. doi.org/10.1142/9789812702593_0060

Shortliffe, E. H. (1976). Computer-Based Medical Consultation: MYCIN. New York: Elsevier. doi.org/10.1016/B978-0-444-00179-5.X5001-X

Sikchi, S. S., Ali, M. S., \& Shkchi, S. (2013). Fuzzy Expert Systems (FES) for Medical Diagnosis. International Journal of Computer Applications, 63(11), pp. 7-16. doi. 10.5120/10508-5466

Siler, W., \& Buckley, J. J. (2005). Fuzzy reasoning fuzzy expert systems and fuzzy reasoning. John Wiley \& Sons, Inc. Hoboken, New Jersey. doi.10.1002/0471698504

Sinclair, D., \& Croskerry, P. (2010). Patient safety and diagnostic error: Tips for your next shift. Canadian Family Physician, 56(1), 28-30. PMID: 20090077

Spiegelhalter, D. J., Myles, J. P., Jones, D. R., \& Abrams, K. R. (1999). An introduction to bayesian methods in health technology assessment. BMJ, 319(7208), pp. 508-512. doi. org/10.1136/bmj.319.7208.508

Steimann, F. (2001). On the use and usefulness of fuzzy sets in medical AI. Artificial Intelligence in Medicine, 21(1-3), pp. 131-137. doi.10.1016/ S0933-3657(00)00077-4

Tan, C. F., Wahidin, L. S., Khalil, S. N., Tamaldin, N., Hu, J., \& Rauterberg, G. W. M. (2016). The application of expert system : a review of research. ARPN Journal of Engineering and Applied Sciences, 11(4), pp. 2448-2453. ISSN 1819-6608

The National Healthcare Statistics Initiative. (2015). National Healthcare Establishment and Workforce Statistics (Hospital) 2012-2013 (1st ed.). Kuala Lumpur: The National Healthcare Statistics Initiative.

Tripathi, K. P. (2011). A Review on knowledge-based expert system : concept and architecture. Artificial Intelligence Techniques - Novel Approaches \& Practical Applications, 4(4), pp. 19-23. doi. 10.5120/2845-226 
Wahabi, H. A., \& Alziedan, R. A. (2012). Reasons behind non-adherence of healthcare practitioners to pediatric asthma guidelines in an emergency department in Saudi Arabia. BMC Health Services Research, 12, pp. 226. doi. 10.1186/1472-6963-12-226.

Wisnivesky, J. P., Lorenzo, J., Lyn-Cook, R., Newman, T., Aponte, A., Kiefer, E., \& Halm, E. a. (2008). Barriers to adherence to asthma management guidelines among inner-city primary care providers. Annals of Allergy, Asthma \& Immunology: Official Publication of the American College of Allergy, Asthma, \& Immunology, 101(3), 264-270. doi. 10.1016/ S1081-1206(10)60491-7.

Yang, C. L., Simons, E., Foty, R. G., Subbarao, P., To, T., \& Dell, S. D. (2016). Misdiagnosis of asthma in schoolchildren. Pediatric Pulmonology, 52(3), pp. 293-302.

Zadeh, L. A. (1965). Fuzzy Sets. Information and Control. 8(1), pp. 338--53. doi: $10.1002 /$ ppul.23541.

Zadeh, L. A. (1972). A fuzzy-set-theoretic interpretation of linguistic hedges. Journal of Cybernetics, 2(3), pp. 4-34. doi. org/10.1080/01969727208542910

Zadeh, L. A. (1988). Fuzzy logic. Computer, 21(4), pp. 83-93. doi:10.1109/2.53

Zarandi, M. H. F., Zolnoori, M., Moin, M., \& Heidarnejad, H. (2010). A fuzzy rule-based expert system for diagnosing asthma. Scientia Iranica Transaction E-Industrial Engineering, 17(2), pp. 129-142. Retrieved from wos:000287001600005

Zolnoori, M., Fazel Zarandi, M. H., Moin, M., \& Taherian, M. (2012). Fuzzy rule-based expert system for evaluating level of asthma control. Journal of Medical Systems, 36(5), pp. 2947-2958. doi. 10.1007/s10916-0119773-3

Zolnoori, M., Hossein, M., Zarandi, F., \& Moin, M. (2010). Fuzzy rule-based expert system for evaluation possibility of fatal asthma. Journal of Health Informatics in Developing Countries, 5(1), pp. 171-184.

Zolnoori, M., Zarandi, M. H. F., \& Moin, M. (2012). Application of intelligent systems in asthma disease: Designing a fuzzy rule-based system for evaluating level of asthma exacerbation. Journal of Medical Systems, 36(4), pp. 2071-2083. doi: 10.1007/s10916-011-9671-8. 